\title{
Knowledge of mother-to-child transmission of HIV by pregnant women in Maputo City, Mozambique
}

Rosa M. Manjate ${ }^{1,2,3}$, Osvaldo F.A. Loquiha ${ }^{4}$, Acácio J.D.E. Sabonete ${ }^{5}$, Khátia R. Munguambe ${ }^{6}$, Julie Cliff', Olivier Degomme ${ }^{1}$, Marleen Temmerman ${ }^{1,8}$, Mohsin Sidat $^{7}$

${ }^{1}$ Ghent University, Ghent, Belgium

${ }^{2}$ Eduardo Mondlane University, Maputo, Mozambique

${ }^{3}$ Ministry of Health, Mozambique

${ }^{4}$ Department of Mathematics and Informatics, Faculty of Sciences, Eduardo Mondlane University, Maputo, Mozambique

${ }^{5}$ National Institute of Health - Ministry of Health, Mozambique

${ }^{6}$ Department of Community Health, Faculty of Medicine, Eduardo Mondlane University, Maputo, Mozambique

${ }^{7}$ Faculty of Medicine, Eduardo Mondlane University, Maputo, Mozambique

${ }^{8}$ Aga Khan University Hospital, Nairobi, Kenya

\begin{abstract}
Introduction: Good knowledge of prevention of mother-to-child transmission (PMTCT) can improve mothers' behavior in seeking care. This study aimed to assess knowledge on PMTCT, human immunodeficiency virus (HIV), and acquired immunodeficiency syndrome (AIDS) at three public antenatal clinics in Maputo City.

Material and methods: Between December 2013 and November 2014, an antenatal care (ANC)/PMTCT cross-sectional exit survey among pregnant women was conveniently conducted. Face-to-face interviews, adjusted odds ratios, and 95\% confidence intervals were applied.

Results: In total, 420 pregnant women were enrolled into the study, and $72.7 \%$ had high PMTCT knowledge, $72 \%$ knew mother-to-child transmission (MTCT) can occur in pregnancy, $76.4 \%$ through breastfeeding, $82.9 \%$ in labor, and $92.7 \%$ knew drugs to reduce MTCT. Similarly, $83.4 \%$ had high HIV prevention knowledge. PMTCT knowledge was more likely to increase with age (AOR $=3.83$; $95 \% \mathrm{CI}$ : $1.36-10.81$ ) and education ( $\mathrm{AOR}=2.16 ; 95 \% \mathrm{CI}$ : 1.15-4.08). HIV prevention knowledge was likely to increase with education ( $\mathrm{AOR}=4.71 ; 95 \% \mathrm{CI}: 1.54-14.36)$, being married ( $\mathrm{AOR}=2.66 ; 95 \% \mathrm{CI}: 1.30-5.43)$, and through condom use ( $\mathrm{AOR}=2.16$; $95 \% \mathrm{CI}: 1.13-4.14)$.
\end{abstract}

Conclusions: Most pregnant women had high PMTCT and HIV prevention knowledge. The youngest, single, and illiterate women have challenges to access information. PMTCT education in verbal mother tongue is required due to limited literacy. Male partner involvement should be strongly supported.

HIV AIDS Rev 2020; 19, 2: 106-115 DOI: https://doi.org/10.5114/hivar.2020.96507

Key words: knowledge, pregnant women, HIV/AIDS, Mozambique, MTCT.

Address for correspondence: Dr. Rosa M. Manjate, Ghent University, Eduardo Mondlane University and Ministry of Health, e-mail: marlene.cuco@gmail.com
Article history:

Received: 08.04.2019

Received in revised form: 06.06.2019

Accepted: 07.07.2019

Available online: 19.06 .2020
International Journal of Hiv-Related Problems

HIV \& AIDS R e v i e w 


\section{Introduction}

Human immunodeficiency virus (HIV)/acquired immune deficiency syndrome (AIDS) epidemic continues to be a challenge for pregnant women and their children, particularly in sub-Saharan Africa (SSA) [1, 2]. In 2017, worldwide, there were 36.9 million of people living with HIV, with 1.4 million pregnant women among them [3] and 1.8 million children under 15 years [4]. Overall, the number of new HIV infections in children decreased by $35 \%$ from 2010 to 2017 . Nevertheless, in 2017 , approximately $70 \%$ of pregnant women and $90 \%$ of 110,000 newly HIV-infected children were in the African region [3, 5]. Over $90 \%$ of HIV infections in children occur through mother-to-child transmission (MTCT) [3]. In the absence of any interventions, MTCT contributes with $15 \%$ to $45 \%$ of child acquired HIV infection, with the lowest risk in developed countries and the highest in developing countries [6]. The chance of HIV MTCT is estimated to be $10 \%$ during pregnancy, $15 \%$ during delivery, and $20 \%$ through breastfeeding $[3,7]$.

Mozambique is a South African country (SAA), which is greatly affected by HIV/AIDS. In 2015, the Mozambican Malaria, HIV/AIDS, and Immunization Indicator Survey (EMASIDA, 2015) found that adult HIV prevalence was 13.2\%. Among 2.1 million Mozambicans living with HIV/ AIDS, $60 \%$ were women [8], and HIV/AIDS prevalence among pregnant women was estimated at $15.8 \%$, varying between $1.1 \%$ and $34 \%$ at ANC sites $[9,10]$. According to national policy, prevention of mother-to-child transmission (PMTCT) is integrated with antenatal care (ANC) [11]. Currently, over $90 \%$ of pregnant women have access to PMTCT interventions. However, the MTCT rate was estimated at $17 \%$ in 2013 and 14\% in 2017 [12], and new pediatric HIV infections decreased only by $42 \%$ from 2010 to 2017 [13], a slower decline than that observed in other countries $[5,13]$.

To support countries greatly affected by HIV/AIDS, the World Health Organization (WHO) strategy of virtual elimination of pediatric HIV infection recommended primary prevention of HIV infection among women of reproductive age, unintended pregnancies among women living with HIV/ AIDS, PMTCT, provision of care, and support to mothers living with HIV/AIDS, their children, and families [14]. In 2016, in an effort to significantly curb HIV/AIDS infections, the Mozambique government, along with others world leaders, committed to the implementation of the UNAIDS 2014 fast-track strategy to ensure that $90 \%$ of pregnant women know their HIV status, $90 \%$ have an access to ART, $90 \%$ of those under treatment have viral suppression, zero new HIV infections among children, and zero stigma by 2020 [2]. These interventions have resulted in a reduction of MTCT to less than 5\% of pregnancies, predominantly in middle- and high-income countries [3].

In some resource constrained SSA countries, obstacles to successful prevention of MTCT/PMTCT prevail. In a study comparing demographic and health survey databases from Mozambique, Uganda, Congo, and Nigeria, Mozambique was placed second, with $69.4 \%$ of pregnant women tested for HIV in ANCs compared to $81.5 \%$ in Nigeria. Overall, PMTCT uptake was lower among less educated women and those with limited knowledge of HIV [15]. Similarly, a South African study found that adolescence, lower education, and late first ANC visits were associated with high PMTCT dropout [16]. The 2017 Mozambican census revealed that $46.3 \%$ of girls between 15 and 25 years old were unable to read and write in any language [17], and almost half of girls are first time pregnant before 20 years old [8]. The Mozambique's PMTCT strategy includes HIV awareness and education, universal access to combined anti-retroviral therapy (cART), institutional birth, and exclusive breastfeeding [12]. The EMASIDA 2015 study observed that in Maputo City, only $61.2 \%$ of women knew that MTCT can occur through breastfeeding and were aware of special drugs taken by pregnant woman to reduce the risk of HIV in MTCT. Sixty-eight percent of women knew that limiting sexual relationships to a single non-infected partner and using condoms with occasional sex partners reduce the risk of HIV infection [8].

Knowledge of prevention of mother-to-child transmission of HIV infection (PMTCT) can improve mother's behavior in seeking PMTCT services [18]. Mozambique PMTCT knowledge data in pregnancy is still limited. This study aimed to assess the knowledge about PMTCT and HIV, and related factors in pregnant women at public antenatal clinics in the capital of Mozambique. These study findings will provide useful information for policy makers and healthcare providers to continually improve ANC/ PMTCT uptake in pregnant women.

\section{Material and methods \\ Study design, population, and study settings}

This survey is a part of a larger study intended to measure adherence to cART for PMTCT. It was an exit healthcenter-based cross-sectional study involving pregnant women attending ANC/PMTCT, carried out from December 2013 to November 2014 in Mavalane health zone, Maputo City. Public primary healthcare in Maputo City has 16 health centers divided into three health zones, according to the referral general hospitals namely Mavalane, Jose Macamo, and Chamanculo. Mavalane health zone is the largest area, comprised of eight public health centers, which provide roughly $50 \%$ of ANC/ PMTCT care in Maputo City. In 2014, Mavalane health zone included $40 \%(980,000)$ of the Maputo City population and $50 \%(61,289)$ of expected pregnancies [19]. The overall HIV prevalence among pregnant women in the southern region of Mozambique where Maputo City is located was estimated at $24 \%$ [9].

\section{Questionnaire design}

The questionnaire was specifically developed for this study in Portuguese, the official language of Mozambique. Questions were taken from the national survey of prevalence, behavioral risks, and information on HIV and AIDS in Mo- 
zambique [20] and completed with few specific questions. Questions intended to assess mother-to-child transmission of HIV routes and prevention knowledge, HIV/AIDS prevention knowledge and related factors such as age, education, marital status, ant-natal attendance, sexual behavior, and practices. Three health technicians supervised by the principal investigator collected the data. Prior to the study, two interactive sessions including study subject's privacy, confidentiality, informed consent, questionnaire contents, interviews procedures, data collection, and quality control were carried out with all interviewers. A pilot test was done among 32 pregnant women attending ANC/PMTCT in Malhangalene and Polana Cimento health centers, also located at Mavalane health zone. Results from the pilot testing were used to improve question clarity, understanding, and consistency, with minor changes made after the pilot test.

\section{Sample size estimation}

The sample size was calculated based on a single proportion formula assuming that $50 \%$ of pregnant women would have a high knowledge of PMTCT of HIV, with $5 \%$ of precision, $95 \%$ of confidence, and $10 \%$ of margin of no response.

\section{Sampling procedures}

From eight operating public health centers in Mavalane health zone, we conveniently selected three, namely Mavalane (MHC), Primeiro de Maio (PMHC), and Primeiro de Junho (PJHC) health centers due to longstanding integrated ANC/PMTCT services. Sample size was proportionally allocated taking into account the total number of pregnant women who attended ANC/PMTCT services in the year previous to the study in each health center to accomplish the total sample required. For study enrolment, pregnant women were approached on their exit from ANC/PMTCT services, informed about the study, and assessed for eligibility. Pregnant women who met the inclusion criteria, namely aged 18 years and older and six months pregnant, were invited to participate. The total of 423 pregnant women were selected from PMHC $(60 \%, 254), \operatorname{MHC}(25 \%, 106)$, and PJHC $(15 \%, 63)$. At health centers, all pregnant women benefit from free integrated ANC/PMTCT package, including ANC/PMTCT education essentially provided at ANC service, mostly delivered by mother and child health nurses $(\mathrm{MCH})$, with assistance of lay counsellors, using a mixture of Portuguese and local languages. Some HIV/AIDS information is also provided through mass media.

\section{Operational definitions}

\section{PMTCT knowledge and HIV prevention knowledge}

MTCT awareness was assessed as whether pregnant women had ever heard about MTCT of HIV. The level of PMTCT knowledge and level of HIV prevention knowledge were defined as two composite outcomes assessed through a set of items or questions regarding MTCT and HIV/AIDS. For PMTCT knowledge, a discrete score was obtained adding up the responses ( 1 for correct answer and 0 otherwise) for awareness of PMTCT of HIV during pregnancy, delivery and through breastfeeding, and the use of drugs to reduce the risk of PMTCT of HIV. The sum of scores for PMTCT knowledge ranged from 0 to 4 , accordingly to correct answers. The level of PMTCT knowledge was defined by categorizing the scores as (0 to 1) expressing low, (2) moderate, and (3 to 4 ) high knowledge on PMTCT.

\section{HIV prevention knowledge}

HIV awareness was assessed as whether pregnant women had ever heard about HIV. For HIV prevention knowledge, a discrete score was obtained summing the binary codes ( 0 and 1 ) for awareness of protective factors against HIV infection (having a single negative sexual partner, condom use with occasional sexual partners, and sexual abstinence), with rejecting myths regarding risk factors for HIV infection (such as mosquito bites and eating together with HIV-infected people). The level of HIV prevention knowledge was categorized with values ranging from 1 (low) to 5 (high knowledge) of correct answers, and further grouped as (1 and 2) expressing low, (3) moderate, and (4 and 5) high knowledge. This choice of cut-off values for both PMTCT and HIV knowledge was preferred not only because it results in a symmetric definition of level of knowledge, but also because it reduces data sparsely.

\section{Data collection}

At ANC/PMTCT exit, face-to-face interviews were carried out to all selected pregnant women who met the inclusion criteria. Data collection was done using a pretested structured questionnaire comprised of 30 questions. The questionnaire was administered in a private room using either Portuguese or whenever necessary, direct translation to Changana, the most spoken native language in Maputo City. Time spent for completion varied, but on average, it was forty-five minutes.

\section{Data analysis}

The data was entered into Epi info version 3.5.5, cleaned and analyzed in SAS/STAT PROC NLMIXED (version 9.4). Two-way contingency tables were used to summarize the data with the Cochran-Mantel-Haenszel test applied to investigate associations between levels of PMTCT and HIV/AIDS knowledge and pregnant woman's socio-demographic characteristics. The bivariate Dale model was used to estimate the joint distribution of levels of MTCT and HIV/AIDS knowledge, while adjusting for effects of covariates [20]. For this model, parameter estimates were expressed in odds ratios and interpreted using ordinal logistic regression models. The model estimation was done in SAS/STAT PROC NLMIXED (version 9.4), using a macro developed by McMillan and Hanson [21]. For inference, $p$-values, and confidence intervals (CI) were calculated for adjusted odds ratios, with significance level set at 5\%. 
The dependence between the outcomes was modelled using the global cross-ratio (GCR), an association measure for contingency tables, for outcomes with 3 or more ordered levels.

\section{Ethical clearance}

Ethical approval was obtained from the Mozambican National Bioethics Committee (Reference no: 357/CNB/12; IRB 00002657). Administrative permit was obtained from the Maputo City Health Directorate and from each health center participating in the study. Oral informed consent was obtained from each enrolled pregnant woman after clear explanation of the study aim and the right to withdraw at any stage, with no negative consequences. Data confidentiality was ensured. All collected data was kept anonymous with access restricted to the research team. Findings were presented as aggregate data with no identifiers.

\section{Results}

Table 1 summarizes socio-demographic features of 420 pregnant women enrolled in the study. The response rate was $99.2 \%$. The majority of pregnant women $(72.3 \%)$ were married or in marital union, $64 \%$ were under 30 years old, with half between 18 and 24 years old, 54.5\% had either primary or no education with limited Portuguese speaking, reading, and writing capabilities, and $46.2 \%$ were unaware of their HIV sero-status.

Table 2 shows the frequency of distribution of motherto-child transmission, with HIV routes and HIV prevention knowledge. The majority of pregnant women (80.4\%) were aware of MTCT, and almost 75\% knew MTCT of HIV can occur during pregnancy, in labor, and through breastfeeding. All pregnant women (100\%) were aware of HIV, and over $90 \%$ knew the three safe sexual practices to reduce HIV infection. Moreover, $82.1 \%$ correctly disagreed that HIV infection can be transmitted through mosquito bites and 97.5\% by sharing food with someone who has HIV.

Table 3 illustrates the results of cross tabulation of PMTCT level and HIV prevention knowledge. Among pregnant women who participated in the study, $72.7 \%$ had high PMTCT knowledge, meaning that they knew all three MTCT of HIV transmission routes and drugs to prevent it, and $83.4 \%$ had high HIV prevention knowledge, meaning that they knew three sexual prevention means and rejected the two most common misconceptions. Only $62.3 \%$ had both high PMTCT and high HIV prevention knowledge.

Table 4 describes a two-way tabulation of socio-demographic characteristics associated with PMTCT and HIV prevention knowledge. PMTCT knowledge was more likely to increase with age $(p=0.040)$, education level $(p=0.035)$, condom use in the last sexual intercourse $(p=0.048)$, and HIV sero-status $(p=0.015)$. The level of HIV prevention knowledge was likely to increase with educational level $(p=0.002)$, marital status $(p=0.004)$, antenatal visits $(p=0.004)$, private counselling session $(p=0.044)$, dis- cussed HIV status with partner $(p=0.001)$, and condom use in the last sexual intercourse $(p=0.006)$.

Table 5 represents results of the bivariate Dale model. The odds of high PMTCT knowledge for pregnant women aged 35 years or more was almost four times that of women between 18-24 years old (AOR $=3.8$; 95\% CI: 1.36-10.81). The odds of high PMTCT knowledge for women with secondary or higher education was also almost three times that of women with no education ( $\mathrm{AOR}=2.8$; $95 \% \mathrm{CI}$ : 1.14-7.08). Regarding HIV prevention, the odds of high knowledge for women with secondary or higher education was almost five times that of women with no education $(\mathrm{AOR}=4.71 ; 95 \% \mathrm{CI}$ : 1.54-14.36). Also, the odds of having high HIV prevention knowledge among married women or living with a partner was almost three times that of a single, widow, or divorced women $(\mathrm{AOR}=2.66 ; 95 \% \mathrm{CI}: 1.30-5.43)$. A similar trend was observed between women who reported condom use and those who did not $(\mathrm{AOR}=2.16$; 95\% CI: 1.13-14).

\section{Discussion}

High levels of knowledge of PMTCT of HIV can improve mother's behavior in seeking care and contribute

Table 1. Socio-demographics characteristics, behavior, and practices of pregnant women at antenatal care (ANC)/prevention of mother-to-child transmission (PMTCT) clinics

\begin{tabular}{|c|c|}
\hline Parameter & $N=420(\%)$ \\
\hline \multicolumn{2}{|l|}{ Age groups } \\
\hline $18-24$ & $132(31.4)$ \\
\hline $25-29$ & $137(32.6)$ \\
\hline $30-34$ & $112(26.7)$ \\
\hline$>35$ & $39(9.3)$ \\
\hline \multicolumn{2}{|l|}{ Education level } \\
\hline No education & $31(7.4)$ \\
\hline Primary & $198(47.1)$ \\
\hline Secondary or higher & $191(45.5)$ \\
\hline \multicolumn{2}{|l|}{ Religion } \\
\hline Christian/Islam & $58(13.8)$ \\
\hline Evangelic & $333(79.3)$ \\
\hline None & $29(6.9)$ \\
\hline \multicolumn{2}{|l|}{ Marital status } \\
\hline Single/widow/divorced & $116(27.7)$ \\
\hline Married/marital union & $303(72.3)$ \\
\hline \multicolumn{2}{|l|}{ HIV status } \\
\hline Positive & $139(33.1)$ \\
\hline Negative & $87(20.7)$ \\
\hline Unknown & $194(46.2)$ \\
\hline \multicolumn{2}{|l|}{ ANC visits } \\
\hline$\leq 3$ & $357(85.0)$ \\
\hline$\geq 4$ & $63(15.0)$ \\
\hline
\end{tabular}


Table 2. HIV/AIDS and prevention of mother-to-child transmission (PMTCT) awareness, and prevention knowledge, among pregnant women at antenatal care (ANC)/PMTCT clinics

\begin{tabular}{|c|c|}
\hline Question & $N=420(\%)$ \\
\hline \multicolumn{2}{|c|}{ Human immunodeficiency virus } \\
\hline \multicolumn{2}{|c|}{ Have you ever heard about HIV/AIDS? } \\
\hline Yes & $420(100)$ \\
\hline No & 0 \\
\hline \multicolumn{2}{|c|}{ Do you know anyone living with HIV/AIDS? } \\
\hline Yes & $295(72.5)$ \\
\hline No & $112(27.5)$ \\
\hline \multicolumn{2}{|c|}{ Pregnant women HIV status } \\
\hline Positive & $139(33.1)$ \\
\hline Negative & $87(20.7)$ \\
\hline Unknown & $194(46.2)$ \\
\hline \multicolumn{2}{|c|}{ Private counseling session for HIV } \\
\hline Yes & $326(78.9)$ \\
\hline No & $87(21.1)$ \\
\hline \multicolumn{2}{|c|}{ Discussed HIV status with partner } \\
\hline Yes & $335(81.1)$ \\
\hline No & $78(18.9)$ \\
\hline \multicolumn{2}{|c|}{ Condom use in the last sexual intercourse } \\
\hline Yes & $268(68.9)$ \\
\hline No & $121(31.1)$ \\
\hline
\end{tabular}

\section{Question} $N=420(\%)$

A person cannot be infected by sharing food with someone who have HIV

\begin{tabular}{l|c}
\hline Yes & $10(2.5)$ \\
\hline No & $392(97.5)$ \\
\hline
\end{tabular}

Limiting sexual relationship to a single not infected partner and condom use occasional partners reduce the risk of getting HIV infection

\begin{tabular}{l|c}
\hline Yes & $325(85.5)$ \\
\hline No & $37(8.8)$ \\
\hline
\end{tabular}

Mother-to-child transmission of HIV

HIV can be transmitted from mother-to-child

\begin{tabular}{l|c}
\hline Yes & $351(80.4)$ \\
\hline No & $69(19.6)$ \\
\hline
\end{tabular}

HIV can be transmitted from mother-to-child during pregnancy

\begin{tabular}{l|l}
\hline Yes & $302(72.0)$ \\
\hline No & $118(28.0)$
\end{tabular}

HIV can be transmitted from mother-to-child during labor

\begin{tabular}{l|c}
\hline Yes & $348(82.9)$ \\
\hline No & $72(17.1)$ \\
\hline
\end{tabular}

HIV can be transmitted from mother-to-child through breast-feeding

\begin{tabular}{l|c}
\hline Yes & $322(76.4)$ \\
\hline No & $99(23.6)$ \\
\hline
\end{tabular}

The risk of MTCT can be reduced if mother take special medicines during pregnancy

\begin{tabular}{l|c}
\hline Yes & $415(92.7)$ \\
\hline No & $5(2.3)$ \\
\hline
\end{tabular}

HIV can be transmitted from mother-to-child through breast-feeding and the risk reduced by special medicines taken in pregnancy

\begin{tabular}{l|c}
\hline Yes & $324(77.1)$ \\
\hline No & $88(21.0)$
\end{tabular}

It is possible to reduce the risk of getting HIV infection through sexual abstinence?

\begin{tabular}{l|c}
\hline Yes & $178(90.0)$ \\
\hline No & $21(10.0)$ \\
\hline
\end{tabular}

HIV infection cannot be transmitted through mosquito bite

\begin{tabular}{l|c}
\hline Yes & $59(14.6)$ \\
\hline No & $345(82.1)$ \\
\hline
\end{tabular}

Table 3. Cross tabulation of level of prevention of mother-to-child transmission (PMTCT) and HIV prevention knowledge in pregnant women at antenatal care (ANC)/PMTCT clinics

\begin{tabular}{l|l|c|c|c|c}
\hline \multicolumn{2}{c|}{} & \multicolumn{4}{|c}{ HIV prevention knowledge } \\
\cline { 3 - 6 } \multicolumn{2}{c|}{} & Low (\%) & Moderate (\%) & High (\%) & Total (\%) \\
\hline \multirow{2}{*}{$\begin{array}{l}\text { PMTCT } \\
\text { knowledge }\end{array}$} & Low (\%) & $1(0.27)$ & $17(4.55)$ & $47(12.57)$ & $65(17.38)$ \\
\cline { 2 - 5 } & Moderate (\%) & $2(0.53)$ & $3(0.80)$ & $32(8.56)$ & $37(9.89)$ \\
\cline { 2 - 6 } & High (\%) & $5(1.34)$ & $34(9.09)$ & $233(62.30)$ & $272(72.73)$ \\
\cline { 2 - 6 } & Total (\%) & $8(2.14)$ & $54(14.44)$ & $312(83.42)$ & $374(100.00)$ \\
\hline
\end{tabular}


Table 4. Socio-demographics characteristics associated with prevention of mother-to-child transmission (PMTCT) and HIV/AIDS knowledge among pregnant women at antenatal care (ANC)/PMTCT clinics

\begin{tabular}{|c|c|c|c|c|c|c|c|c|c|c|}
\hline \multirow[t]{2}{*}{ Parameter } & \multicolumn{5}{|c|}{ PMTCT knowledge } & \multicolumn{5}{|c|}{ HIV/AIDS knowledge } \\
\hline & $\begin{array}{l}\text { Low } \\
\text { (\%) }\end{array}$ & $\begin{array}{c}\text { Moderate } \\
\text { (\%) }\end{array}$ & $\begin{array}{l}\text { High } \\
(\%)\end{array}$ & $\begin{array}{l}\text { Total } \\
\text { (\%) }\end{array}$ & $p$-value & $\begin{array}{l}\text { Low } \\
\text { (\%) }\end{array}$ & $\begin{array}{c}\text { Moderate } \\
\text { (\%) }\end{array}$ & $\begin{array}{l}\text { High } \\
(\%)\end{array}$ & $\begin{array}{l}\text { Total } \\
\text { (\%) }\end{array}$ & $p$-value \\
\hline \multicolumn{11}{|l|}{ Age groups } \\
\hline $18-24$ & $\begin{array}{c}24 \\
(6.14)\end{array}$ & $\begin{array}{c}17 \\
(4.35)\end{array}$ & $\begin{array}{c}78 \\
(19.95)\end{array}$ & $\begin{array}{c}119 \\
(30.43)\end{array}$ & \multirow[t]{4}{*}{0.0408} & $\begin{array}{c}3 \\
(0.79)\end{array}$ & $\begin{array}{c}15 \\
(3.93)\end{array}$ & $\begin{array}{c}98 \\
(25.65)\end{array}$ & $\begin{array}{c}116 \\
(30.37)\end{array}$ & \multirow[t]{4}{*}{0.9967} \\
\hline $25-29$ & $\begin{array}{c}22 \\
(5.63)\end{array}$ & $\begin{array}{c}10 \\
(2.56)\end{array}$ & $\begin{array}{c}97 \\
(24.81)\end{array}$ & $\begin{array}{c}129 \\
(32.99)\end{array}$ & & $\begin{array}{c}3 \\
(0.79)\end{array}$ & $\begin{array}{c}21 \\
(5.50)\end{array}$ & $\begin{array}{c}102 \\
(26.70)\end{array}$ & $\begin{array}{c}126 \\
(32.98)\end{array}$ & \\
\hline $30-34$ & $\begin{array}{c}17 \\
(4.35)\end{array}$ & $\begin{array}{c}10 \\
(2.56)\end{array}$ & $\begin{array}{c}79 \\
(20.20)\end{array}$ & $\begin{array}{c}106 \\
(27.11)\end{array}$ & & $\begin{array}{c}0 \\
(0.0)\end{array}$ & $\begin{array}{c}14 \\
(3.66)\end{array}$ & $\begin{array}{c}91 \\
(23.82)\end{array}$ & $\begin{array}{c}105 \\
(27.49)\end{array}$ & \\
\hline$>35$ & $\begin{array}{c}3 \\
(0.77)\end{array}$ & $\begin{array}{c}3 \\
(0.77)\end{array}$ & $\begin{array}{c}31 \\
(7.93)\end{array}$ & $\begin{array}{c}37 \\
(9.46)\end{array}$ & & $\begin{array}{c}2 \\
(0.52)\end{array}$ & $\begin{array}{c}5 \\
(1.31)\end{array}$ & $\begin{array}{c}28 \\
(7.33)\end{array}$ & $\begin{array}{c}35 \\
(9.16)\end{array}$ & \\
\hline \multicolumn{11}{|l|}{ Education level } \\
\hline No education & $\begin{array}{c}9 \\
(2.30)\end{array}$ & $\begin{array}{c}2 \\
(0.51)\end{array}$ & $\begin{array}{c}19 \\
(4.86)\end{array}$ & $\begin{array}{c}30 \\
(7.67)\end{array}$ & \multirow[t]{3}{*}{0.0355} & $\begin{array}{c}1 \\
(0.26)\end{array}$ & $\begin{array}{c}7 \\
(1.83)\end{array}$ & $\begin{array}{c}23 \\
(6.02)\end{array}$ & $\begin{array}{c}31 \\
(8.12)\end{array}$ & \multirow[t]{3}{*}{0.002} \\
\hline Primary & $\begin{array}{c}34 \\
(8.70)\end{array}$ & $\begin{array}{c}20 \\
(5.12)\end{array}$ & $\begin{array}{c}132 \\
(33.76)\end{array}$ & $\begin{array}{c}186 \\
(47.57)\end{array}$ & & $\begin{array}{c}5 \\
(1.31)\end{array}$ & $\begin{array}{c}34 \\
(8.90)\end{array}$ & $\begin{array}{c}143 \\
(37.43)\end{array}$ & $\begin{array}{c}182 \\
(47.64)\end{array}$ & \\
\hline Secondary or higher & $\begin{array}{c}23 \\
(5.88)\end{array}$ & $\begin{array}{c}18 \\
(4.60)\end{array}$ & $\begin{array}{c}134 \\
(34.27)\end{array}$ & $\begin{array}{c}175 \\
(44.76)\end{array}$ & & $\begin{array}{c}2 \\
(0.52)\end{array}$ & $\begin{array}{c}14 \\
(3.66)\end{array}$ & $\begin{array}{c}153 \\
(40.05)\end{array}$ & $\begin{array}{c}169 \\
(44.24)\end{array}$ & \\
\hline \multicolumn{11}{|l|}{ Religion } \\
\hline Christian/Islam & $\begin{array}{c}9 \\
(2.30)\end{array}$ & $\begin{array}{c}5 \\
(1.28)\end{array}$ & $\begin{array}{c}41 \\
(10.49)\end{array}$ & $\begin{array}{c}55 \\
(14.07)\end{array}$ & \multirow[t]{3}{*}{0.8359} & $\begin{array}{c}1 \\
(0.26)\end{array}$ & $\begin{array}{c}8 \\
(2.09)\end{array}$ & $\begin{array}{c}45 \\
(11.78)\end{array}$ & $\begin{array}{c}54 \\
(14.14)\end{array}$ & \multirow[t]{3}{*}{0.259} \\
\hline Evangelic & $\begin{array}{c}52 \\
(13.30) \\
\end{array}$ & $\begin{array}{c}32 \\
(8.18)\end{array}$ & $\begin{array}{c}223 \\
(57.03)\end{array}$ & $\begin{array}{c}307 \\
(78.52)\end{array}$ & & $\begin{array}{c}5 \\
(1.31)\end{array}$ & $\begin{array}{c}41 \\
(10.73)\end{array}$ & $\begin{array}{c}254 \\
(66.49)\end{array}$ & $\begin{array}{c}300 \\
(78.53)\end{array}$ & \\
\hline None & $\begin{array}{c}5 \\
(1.28)\end{array}$ & $\begin{array}{c}3 \\
(0.77)\end{array}$ & $\begin{array}{c}21 \\
(5.37)\end{array}$ & $\begin{array}{c}29 \\
(7.42)\end{array}$ & & $\begin{array}{c}2 \\
(0.52)\end{array}$ & $\begin{array}{c}6 \\
(1.57)\end{array}$ & $\begin{array}{c}20 \\
(5.24)\end{array}$ & $\begin{array}{c}28 \\
(7.33)\end{array}$ & \\
\hline \multicolumn{11}{|l|}{ Marital status } \\
\hline $\begin{array}{l}\text { Single/widow/ } \\
\text { divorced }\end{array}$ & $\begin{array}{c}14 \\
(3.59)\end{array}$ & $\begin{array}{c}14 \\
(3.59)\end{array}$ & $\begin{array}{c}79 \\
(20.26)\end{array}$ & $\begin{array}{c}107 \\
(27.44)\end{array}$ & \multirow[t]{2}{*}{0.2931} & $\begin{array}{c}3 \\
(0.79)\end{array}$ & $\begin{array}{c}24 \\
(6.28)\end{array}$ & $\begin{array}{c}73 \\
(19.11)\end{array}$ & $\begin{array}{c}100 \\
(26.18)\end{array}$ & \multirow[t]{2}{*}{0.0042} \\
\hline $\begin{array}{l}\text { Married/marital } \\
\text { union }\end{array}$ & $\begin{array}{c}52 \\
(13.33)\end{array}$ & $\begin{array}{c}26 \\
(6.67)\end{array}$ & $\begin{array}{c}205 \\
(52.56)\end{array}$ & $\begin{array}{c}283 \\
(72.56)\end{array}$ & & $\begin{array}{c}5 \\
(1.31)\end{array}$ & $\begin{array}{c}31 \\
(8.12)\end{array}$ & $\begin{array}{c}246 \\
(64.40)\end{array}$ & $\begin{array}{c}282 \\
(73.82)\end{array}$ & \\
\hline \multicolumn{11}{|l|}{ ANC visits } \\
\hline$\leq 3$ & $\begin{array}{c}61 \\
(15.60)\end{array}$ & $\begin{array}{c}34 \\
(8.70)\end{array}$ & $\begin{array}{c}240 \\
(61.38)\end{array}$ & $\begin{array}{c}335 \\
(85.68)\end{array}$ & \multirow[t]{2}{*}{0.2273} & $\begin{array}{c}4 \\
(1.05)\end{array}$ & $\begin{array}{c}51 \\
(13.35)\end{array}$ & $\begin{array}{c}274 \\
(71.73)\end{array}$ & $\begin{array}{c}329 \\
(86.13)\end{array}$ & \multirow[t]{2}{*}{0.0047} \\
\hline$\geq 4$ & $\begin{array}{c}5 \\
(1.28)\end{array}$ & $\begin{array}{c}6 \\
(1.53)\end{array}$ & $\begin{array}{c}45 \\
(11.51)\end{array}$ & $\begin{array}{c}56 \\
(14.32)\end{array}$ & & $\begin{array}{c}4 \\
(1.05)\end{array}$ & $\begin{array}{c}4 \\
(1.05)\end{array}$ & $\begin{array}{c}45 \\
(11.78)\end{array}$ & $\begin{array}{c}53 \\
(13.87)\end{array}$ & \\
\hline \multicolumn{11}{|l|}{ Private counseling session } \\
\hline No & $\begin{array}{c}8 \\
(2.05)\end{array}$ & $\begin{array}{c}9 \\
(2.31)\end{array}$ & $\begin{array}{c}57 \\
(14.62)\end{array}$ & $\begin{array}{c}74 \\
(18.97)\end{array}$ & \multirow[t]{2}{*}{0.3016} & $\begin{array}{c}4 \\
(1.06)\end{array}$ & $\begin{array}{c}7 \\
(1.85)\end{array}$ & $\begin{array}{c}62 \\
(16.36)\end{array}$ & $\begin{array}{c}73 \\
(19.26)\end{array}$ & \multirow[t]{2}{*}{0.0442} \\
\hline Yes & $\begin{array}{c}57 \\
(14.62)\end{array}$ & $\begin{array}{c}31 \\
(7.95)\end{array}$ & $\begin{array}{c}228 \\
(58.46)\end{array}$ & $\begin{array}{c}316 \\
(81.03)\end{array}$ & & $\begin{array}{c}4 \\
(1.06)\end{array}$ & $\begin{array}{c}47 \\
(12.40)\end{array}$ & $\begin{array}{c}255 \\
(67.28)\end{array}$ & $\begin{array}{c}306 \\
(80.74)\end{array}$ & \\
\hline \multicolumn{11}{|c|}{ Discussed HIV status with partner } \\
\hline No & $\begin{array}{c}14 \\
(3.62)\end{array}$ & $\begin{array}{c}8 \\
(2.07)\end{array}$ & $\begin{array}{c}53 \\
(13.70)\end{array}$ & $\begin{array}{c}75 \\
(19.38)\end{array}$ & \multirow[t]{2}{*}{0.9048} & $\begin{array}{c}3 \\
(0.79)\end{array}$ & $\begin{array}{c}18 \\
(4.75)\end{array}$ & $\begin{array}{c}53 \\
(13.98)\end{array}$ & $\begin{array}{c}74 \\
(19.53)\end{array}$ & \multirow[t]{2}{*}{0.0098} \\
\hline Yes & $\begin{array}{c}52 \\
(13.44) \\
\end{array}$ & $\begin{array}{c}32 \\
(8.27)\end{array}$ & $\begin{array}{c}228 \\
(58.91)\end{array}$ & $\begin{array}{c}312 \\
(80.62)\end{array}$ & & $\begin{array}{c}5 \\
(1.32)\end{array}$ & $\begin{array}{c}37 \\
(9.76)\end{array}$ & $\begin{array}{c}263 \\
(69.39)\end{array}$ & $\begin{array}{c}305 \\
(80.47)\end{array}$ & \\
\hline \multicolumn{11}{|c|}{ Condom use last sexual intercourse } \\
\hline No & $\begin{array}{c}28 \\
(7.65)\end{array}$ & $\begin{array}{c}10 \\
(2.73)\end{array}$ & $\begin{array}{c}77 \\
(21.04)\end{array}$ & $\begin{array}{c}115 \\
(31.42)\end{array}$ & \multirow[t]{2}{*}{0.0487} & $\begin{array}{c}5 \\
(1.40)\end{array}$ & $\begin{array}{c}23 \\
(6.42)\end{array}$ & $\begin{array}{c}84 \\
(23.46)\end{array}$ & $\begin{array}{c}112 \\
(31.28)\end{array}$ & \multirow[t]{2}{*}{0.0063} \\
\hline Yes & $\begin{array}{c}35 \\
(9.56)\end{array}$ & $\begin{array}{c}27 \\
(7.38)\end{array}$ & $\begin{array}{c}189 \\
(51.64)\end{array}$ & $\begin{array}{c}251 \\
(68.58)\end{array}$ & & $\begin{array}{c}3 \\
(0.84)\end{array}$ & $\begin{array}{c}27 \\
(7.54)\end{array}$ & $\begin{array}{c}216 \\
(60.34)\end{array}$ & $\begin{array}{c}246 \\
(68.72)\end{array}$ & \\
\hline
\end{tabular}


Table 4. Cont.

\begin{tabular}{|c|c|c|c|c|c|c|c|c|c|c|}
\hline \multirow[t]{2}{*}{ Parameter } & \multicolumn{5}{|c|}{ PMTCT knowledge } & \multicolumn{5}{|c|}{ HIV/AIDS knowledge } \\
\hline & $\begin{array}{l}\text { Low } \\
\text { (\%) }\end{array}$ & $\begin{array}{c}\text { Moderate } \\
\text { (\%) }\end{array}$ & $\begin{array}{l}\text { High } \\
(\%)\end{array}$ & $\begin{array}{c}\text { Total } \\
\text { (\%) }\end{array}$ & $p$-value & $\begin{array}{l}\text { Low } \\
\text { (\%) }\end{array}$ & $\begin{array}{l}\text { Moderate } \\
\text { (\%) }\end{array}$ & $\begin{array}{l}\text { High } \\
\text { (\%) }\end{array}$ & $\begin{array}{c}\text { Total } \\
\text { (\%) }\end{array}$ & $p$-value \\
\hline \multicolumn{11}{|c|}{ Know people living with HIV/AIDS } \\
\hline No & $\begin{array}{c}51 \\
(13.28)\end{array}$ & $\begin{array}{c}24 \\
(6.25)\end{array}$ & $\begin{array}{c}206 \\
(53.65)\end{array}$ & $\begin{array}{c}281 \\
(73.18)\end{array}$ & \multirow[t]{2}{*}{0.1041} & $\begin{array}{c}7 \\
(1.86)\end{array}$ & $\begin{array}{c}40 \\
(10.64)\end{array}$ & $\begin{array}{c}224 \\
(59.57)\end{array}$ & $\begin{array}{c}271 \\
(72.07)\end{array}$ & \multirow[t]{2}{*}{0.567} \\
\hline Yes & $\begin{array}{c}14 \\
(3.65)\end{array}$ & $\begin{array}{c}16 \\
(4.17)\end{array}$ & $\begin{array}{c}73 \\
(19.01)\end{array}$ & $\begin{array}{c}103 \\
(26.82)\end{array}$ & & $\begin{array}{c}1 \\
(0.27)\end{array}$ & $\begin{array}{c}14 \\
(3.72)\end{array}$ & $\begin{array}{c}90 \\
(23.94)\end{array}$ & $\begin{array}{c}105 \\
(27.93)\end{array}$ & \\
\hline \multicolumn{11}{|l|}{ HIV status } \\
\hline Positive & $\begin{array}{c}15 \\
(3.84)\end{array}$ & $\begin{array}{c}7 \\
(1.79)\end{array}$ & $\begin{array}{c}57 \\
(14.58)\end{array}$ & $\begin{array}{c}79 \\
(20.20)\end{array}$ & \multirow[t]{3}{*}{0.0158} & $\begin{array}{c}2 \\
(0.52)\end{array}$ & $\begin{array}{c}12 \\
(3.14)\end{array}$ & $\begin{array}{c}116 \\
(30.37)\end{array}$ & $\begin{array}{c}130 \\
(34.03)\end{array}$ & \multirow[t]{3}{*}{0.1118} \\
\hline Negative & $\begin{array}{c}11 \\
(2.81)\end{array}$ & $\begin{array}{c}18 \\
(4.60)\end{array}$ & $\begin{array}{c}104 \\
(26.60)\end{array}$ & $\begin{array}{c}133 \\
(34.02)\end{array}$ & & $\begin{array}{c}2 \\
(0.52)\end{array}$ & $\begin{array}{c}9 \\
(2.36)\end{array}$ & $\begin{array}{c}67 \\
(17.54)\end{array}$ & $\begin{array}{c}78 \\
(20.42)\end{array}$ & \\
\hline Unknown & $\begin{array}{c}40 \\
(10.23)\end{array}$ & $\begin{array}{c}15 \\
(3.84)\end{array}$ & $\begin{array}{c}124 \\
(31.71)\end{array}$ & $\begin{array}{c}179 \\
(45.78)\end{array}$ & & $\begin{array}{c}4 \\
(1.05)\end{array}$ & $\begin{array}{c}34 \\
(8.90)\end{array}$ & $\begin{array}{c}136 \\
(35.60)\end{array}$ & $\begin{array}{c}174 \\
(45.55)\end{array}$ & \\
\hline
\end{tabular}

to a decrease in the rate of mother-to-child transmission of HIV [22]. This study assessed PMTCT and HIV prevention knowledge as well as related factors among pregnant women at ANC/PMTCT exit.

The majority of pregnant women $(72.7 \%)$ had a high level of PMTCT knowledge. This was consistent with a hospital-based cross-sectional survey carried out in Kathmandu (Nepal), where $84.4 \%$ of pregnant women knew all three MTCT transmission routes, although knowledge of drugs to prevent it was relatively low [23]. However, the level of PMTCT knowledge found in our study was higher than in two Ethiopian institutional-based surveys in Assosa town (57.5\%) [24] and East Gojjam (52\%) [25], and 48\% at Kiisi referral hospital in Kenya [26]. In our study, we also observed that $77.1 \%$ of pregnant women knew that MTCT can occur through breastfeeding and the existence of drugs to prevent it, compared to $61.2 \%$ who answered correctly in the Mozambican Malaria, HIV/AIDS, and Immunization Indicator Survey (IMASIDA, 2015) [8].

Similarly, the majority of pregnant women $(83.4 \%)$ had a high HIV prevention knowledge. This result was also in line with a Nepali findings [23]. Furthermore, $85.5 \%$ of pregnant women knew that limiting sexual life to a single not infected with HIV partner and condom use with occasional partners, can reduce the risk of HIV infection, as compared to $68 \%$ found in IMASIDA, 2015 [8] and 63.8\% observed at community-based cross-sectional survey in Northeast Ethiopia in Meket [18]. The difference between our findings and IMASIDA, 2015 was expected, since our results were generated from ANC/ PMTCT-based survey, and pregnant women are more likely to have PMTCT and HIV information exposure at ANC clinics [18], while IMASIDA was countrywide population-based survey, covering both pregnant and not pregnant women in rural and urban settings [8].

Consistent with previous results $[18,25]$, pregnant women with secondary or higher education levels were almost three times $(\mathrm{AOR}=2.8 ; 95 \% \mathrm{CI}: 1.14-7.08)$ more likely to have PMTCT knowledge. Similarly, more educated pregnant women were almost five times (AOR $=4.71 ; 95 \% \mathrm{CI}$ : 1.54 14.36) more likely to have high HIV prevention knowledge then their less formally educated counterparts. However, $54.5 \%$ of pregnant women in our sample had either primary level or no formal education. In Maputo City, ANC/ PMTCT/HIV information, both oral and written, is mostly provided in Portuguese, potentially limiting illiterate pregnant women's ability to speak, read, understand, and follow ANC/PMTCT recommendations [27, 28]. Consequently, the higher PMTCT and HIV prevention knowledge could be greatly related to high HIV/AIDS exposure, as 33\% of pregnant women were HIV-positive and $72.5 \%$ knew someone suffering from AIDS.

Although there are slight differences in estimated levels of PMTCT and HIV prevention knowledge, the majority of discussed studies $[24,29,30]$ and our study results underscore the importance of education for women and girls to understand PMTCT and HIV prevention information to fully benefit from health services. As in other settings [18] and in line with education policy in Mozambique, increasing girls' schooling should continue to be amongst priorities. In the case of Mozambique, verbal mother tongue should be used for illiterate women at ANC/ PMTCT to ensure all women benefit from MTCT and HIV/AIDS information and care.

Our findings also revealed that older pregnant women were more likely to have higher PMTCT knowledge $(\mathrm{AOR}=3.8 ; 95 \% \mathrm{CI}: 1.36-10.81)$. As described in a similar Kenyan survey [26], the fear of HIV disclosure and societal judgment surrounding early pregnancies may have restricted access to PMTCT information for younger women in our study. In addition, older women were more likely to have had previous ANC visits and PMTCT information exposure in earlier pregnancies. Finally, current older pregnant women were more likely to have been exposed to information, since they were younger in the onset of HIV 
Table 5. Adjusted odds ratio and $95 \% \mathrm{Cl}$ for factors related to prevention of mother-to-child transmission (PMTCT) and HIV prevention knowledge in pregnant women at antenatal care (ANC)/PMTCT clinics

\begin{tabular}{|c|c|c|c|c|c|c|c|c|}
\hline \multirow[t]{3}{*}{ Parameter } & \multicolumn{4}{|c|}{ PMTCT knowledge } & \multicolumn{4}{|c|}{ HIV prevention knowledge } \\
\hline & \multirow[t]{2}{*}{ O.R. } & \multicolumn{2}{|c|}{$95 \% \mathrm{Cl}$} & \multirow[t]{2}{*}{$p$-value } & \multirow[t]{2}{*}{ O.R. } & \multicolumn{2}{|c|}{$95 \% \mathrm{Cl}$} & \multirow[t]{2}{*}{$p$-value } \\
\hline & & Lower & Upper & & & Lower & Upper & \\
\hline \multicolumn{9}{|l|}{ Age group (years) } \\
\hline $18-24$ & 1.00 & & & & 1.00 & & & \\
\hline $25-29$ & 1.95 & 1.07 & 3.56 & 0.031 & 1.19 & 0.55 & 2.57 & 0.660 \\
\hline $30-34$ & 2.29 & 1.17 & 4.50 & 0.017 & 1.66 & 0.69 & 4.00 & 0.263 \\
\hline$>35$ & 3.83 & 1.36 & 10.81 & 0.011 & 1.04 & 0.35 & 3.08 & 0.948 \\
\hline \multicolumn{9}{|l|}{ Education } \\
\hline No education & 1.00 & & & & 1.00 & & & \\
\hline Primary & 1.40 & 0.59 & 3.30 & 0.448 & 1.24 & 0.47 & 3.30 & 0.666 \\
\hline Secondary or higher & 2.84 & 1.14 & 7.08 & 0.025 & 4.71 & 1.54 & 14.36 & 0.007 \\
\hline \multicolumn{9}{|l|}{ Religion } \\
\hline Christian/Islam & 1.00 & & & & 1.00 & & & \\
\hline Evangelical & 1.03 & 0.51 & 2.06 & 0.939 & 1.19 & 0.49 & 2.88 & 0.708 \\
\hline None & 1.40 & 0.44 & 4.51 & 0.568 & 0.84 & 0.22 & 3.29 & 0.808 \\
\hline \multicolumn{9}{|l|}{ Marital status } \\
\hline Single/widow/divorced & 1.00 & & & & 1.00 & & & \\
\hline Married/marital union & 0.76 & 0.41 & 1.39 & 0.371 & 2.66 & 1.30 & 5.43 & 0.008 \\
\hline \multicolumn{9}{|l|}{ ANC visits } \\
\hline \multicolumn{9}{|l|}{$\leq 3$} \\
\hline$\geq 4$ & 1.73 & 0.77 & 3.89 & 0.186 & 0.86 & 0.34 & 2.15 & 0.746 \\
\hline \multicolumn{9}{|l|}{ Private counseling session } \\
\hline No & 1.00 & & & & 1.00 & & & \\
\hline Yes & 0.49 & 0.24 & 1.02 & 0.056 & 1.49 & 0.64 & 3.49 & 0.360 \\
\hline \multicolumn{9}{|c|}{ Discussed HIV status with partner } \\
\hline No & 1.00 & & & & 1.00 & & & \\
\hline Yes & 1.05 & 0.53 & 2.09 & 0.885 & 1.45 & 0.64 & 3.31 & 0.376 \\
\hline \multicolumn{9}{|c|}{ Condom use last sexual intercourse } \\
\hline No & 1.00 & & & & 1.00 & & & \\
\hline Yes & 1.68 & 0.98 & 2.87 & 0.059 & 2.16 & 1.13 & 4.14 & 0.020 \\
\hline \multicolumn{9}{|l|}{ Know people with HIV/AIDS } \\
\hline No & 1.00 & & & & 1.00 & & & \\
\hline Yes & 0.67 & 0.38 & 1.21 & 0.186 & 0.98 & 0.46 & 2.11 & 0.960 \\
\hline \multicolumn{9}{|l|}{ HIV status } \\
\hline Positive & 1.00 & & & & 1.00 & & & \\
\hline Negative & 0.69 & 0.33 & 1.45 & 0.333 & 0.76 & 0.29 & 1.97 & 0.571 \\
\hline Unknown & 0.61 & 0.32 & 1.16 & 0.131 & 1.02 & 0.44 & 2.36 & 0.955 \\
\hline \multicolumn{9}{|l|}{ Global cross-ratio model } \\
\hline$\Delta$ & 2.03 & 0.39 & 10.7 & 0.403 & & & & \\
\hline$\delta_{1}$ & 0.69 & 0.14 & 3.27 & 0.636 & & & & \\
\hline
\end{tabular}

epidemic, when PMTCT and HIV/AIDS prevention information were more widespread in Maputo City than nowadays.
In the same way, pregnant women who were married or living with a partner were more likely to have high HIV knowledge $(\mathrm{AOR}=2.66 ; 95 \% \mathrm{CI}$ : 1.30-54.43), suggesting 
that open discussion of HIV/AIDS issues with partners have a positive effect on women information, as quite often males are more educated and informed than women, although less likely to attend health facility [31-34]. Both findings pose challenges to the public health sector to improve access to PMTCT/HIV information to younger and single women to benefit from PMTCT/HIV interventions.

The HIV infection rate of $33 \%$ among pregnant women who disclosed their status in our study was higher than Maputo City HIV prevalence estimation of $24 \%$ [9], but similar to that found among pregnant women in Manhica District (34\%) in Maputo Province [10], where Maputo City and Gaza Province (30\%) are located [9]. These very high HIV rates among pregnant women demonstrate the magnitude of HIV/AIDS threat in Mozambique, particularly among women and their children, highlighting the need for more aggressive interventions to dramatically reduce the motherto-child infection rate currently estimated at $14 \%$, despite over $90 \%$ PMTCT services coverage.

On other hand, we found that $46.2 \%$ of pregnant women did not know their HIV sero-status. This could be in part due to long waiting time for HIV results, which could take up to 3 months at the time of this study, or a late HIV test uptake, as the majority of pregnant women had less than three ANC consultations. Although our study did not look into this aspect, some previous findings have also shown fear of stigma and discrimination as factors preventing pregnant women from HIV test uptake and sero-status disclosure [26]. Unknown sero-status is amongst the reasons for late PMTCT enrolment and increasing risk of MTCT of HIV. Thus, another specific study is needed to better characterize the reasons of high rates of unknown HIV sero-status and address interventions accordingly.

Furthermore, a positive association is mostly found between high PMTCT knowledge and a higher ANC visits score $[19,20]$. However, in our study regardless of high PMTCT knowledge, $85 \%$ of pregnant women reported to attend three or fewer ANC visits, suggesting an exposure to PMTCT and HIV information outside ANC/PMTCT. In Mozambique, ANC is the only system for PMTCT to ensure MTCT decrease and pediatric HIV infections reduction. Strategies to increase ANC/PMTCT uptake should be implemented to curb the risk of poor PMTCT outcomes.

\section{Study limitations}

This study had some limitations. The health centers as well as participants were conveniently selected. The facilities included and pregnant attendees may not necessarily be similar to non-public healthcare facilities and other public services, such as neighborhood and rural health local services. Therefore, the results of this study may not reflect the reality within the whole of Maputo City and/or of Mozambique and should be contextualized and interpreted with caution. However, women's friendly environment created at the study sites, confidentiality, the use of subjects' mother tongues, and opportunities provided for discussion contrib- uted to a good relationship between the study subjects and the interviewers, so these results are more likely to accurately represent pregnant woman's opinions.

\section{Conclusions}

The majority of pregnant women in this study indicated high PMTCT and HIV prevention knowledge, and low coverage of four or more ANC visits. Older, more highly educated, and married pregnant women had a better PMTCT and HIV prevention knowledge. The youngest, single, and illiterate still face challenges to access ANC, PMTCT, and HIV information. Additional MTCT education is required and should be delivered in an integrated manner within HIV/AIDS strategies to pregnant women, including verbal communication in mother tongue due to limited literacy. Male partner involvement can yield better PMTCT outcomes.

\section{Recommendations}

These findings suggest provision of PMTCT and HIV information more effectively to younger, illiterate, and single pregnant women. More ANC visits should also be widely encouraged to contribute to PMTCT uptake. A friendly environment, the use of the mother tongue in an interactive communication allowing pregnant women to pose and discuss questions, seems more valuable in contrast to using Portuguese due to low literacy. Strengthening male partner involvement may be a factor to improved PMTCT outcomes. Finally, our study findings are likely to be useful for policy makers and healthcare providers, as they continually strive to improve MTCT education approaches for better PMTCT results.

\section{Acknowledgments}

Authors are grateful to the VRIL-UOS Flemish Interuniversity Council Development Cooperation, Ghent University and Eduardo Mondlane University for funding and technical assistance. We particularly acknowledge the valuable contribution of study subjects and health workers from the study sites. Special thanks to Dr Roxan Hoek for her assistance and to Professor Herman Meullman for his guidance and support.

\section{Conflict of interest}

The authors declare no conflict of interest with respect to the research, authorship, and/or publication of this article.

\section{References}

1. UNAIDS. Joint United Nations Programme on HIV/AIDS. Fasttrack strategy to end the AIDS epidemic by 2030. UNAIDS; 2014. Available from: https://www.unaids.org/en/resources/campaigns/ World-AIDS-Day-Report-2014.

2. UNAIDS. On the fast-track to end AIDS. 2016-2021 Strategy. Geneva: UNAIDS; 2015. Available from: https://aidsfree.usaid.gov/re- 
sources/prevention.udate/edtions/january.2016/unaids.2016-2021 strategy for aids free generation.

3. WHO. Mother-to-child transmission of HIV. WHO; 2018. Available from: https://www.who.int/hiv/topics/mtct/en/.

4. UNAIDS. Joint United Nations Programme on HIV/AIDS. Faststrategy to end HIV/AIDS epidemic in 2030. UNAIDS; 2018 Availble from: https://www.unaids.org/en/resources/campaigns/ World-AIDS-Day-Report-2014.

5. WHO. Prevention of mother-to-child transmission (PMTCT). Global Health Observatory (GHO) data 2018. Available from: https:// www.who.int/gho/hiv/epidemic_response/PMTCT_text/en/Prevention of mother-to-child transmission (PMTCT).

6. WHO. Mother to child transmission of HIV. WHO; 2011. Available from: https://www.who.int/hiv/topics/mtct.

7. Volmink JA, Marais B. HIV: Mother-to-child transmission. BMJ Clin Evid 2008; 2008: 0909.

8. Ministério da Saúde, Inquérito de Indicadores de Imunização, Malária e HIV/SIDA em Moçambique (IMASIDA). Caverton, Maryland, USA:MISAU,INE e ICFI 2015. Available from: www. dhsprogram.com/pubs/pdf/AIS12/AIS12-SP.pdf.

9. INS, I.N.d.S., I.N.d.E. INE, and G.T.M.d.C.a.H.S. GTM, Ronda de Vigilância Epidemiológica do HIV e sífilis em Mulheres Grávidas em Moçambique: Principais Resultados; Maputo, Moçambique 2011. Available from: www.misau.gov.mz.

10. Rupérez M, González R, Maculuve S, et al. Maternal HIV infection is an important health determinant in non-HIV-infected infants. AIDS 2017; 31: 1545-1553.

11. Ministério da Saúde, Plano Nacional de Eliminação da Transmissão Vertical, Moçambique 2012-2015; 2012.

12. UNAIDS. Spectrum 2.57; 2017.

13. UNAIDS. UNAIDS data 2018 | UNAIDS. 2018. Available from: https://www.unaids.org/en/resources/documents/2018/unaidsdata-2018.

14. UNAIDS. Global Plan towards the Elimination of New HIV infection among Children by 2015 and Keeping Their Mothers Alive, 2011-2015. Geneva: Joint Uited Nations Programme on HIV/ AIDS; 2011.

15. Gunn JKL, Asaolu IO, Center KE, et al. Antenatal care and uptake of HIV testing among pregnant women in sub-Saharan Africa: a cross-sectional study. J Int AIDS Soc 2016; 19: 20605.

16. Woldesenbet S, Jackson D, Lombard C, et al. Missed opportunities along the prevention of mother-to-child transmission services cascade in South Africa: uptake, determinants, and attributable risk. PLoS One 2015; 10: e0132425.

17. Instituto Nacional de Estatística. IV Recenseamento Geral da População e Habitação de 2017.

18. Birhane T, Tessema GA, Alene KA, Dadi AF. Knowledge of pregnant women on mother-to-child transmission of HIV in Meket district, Northeast Ethiopia. J Pregnancy 2015; 2015: 960830.

19. Instituto Nacional de Estatística. III recenseamento geral da população e habitação, 2007.

20. Dale JR. Global cross-ratio models for bivariate, discrete, ordered responses. Biometrics 1986; 42: 909-917.

21. McMillan G, Hanson T. SAS macro BDM for fitting the Dale regression model to bivariate ordinal response data. Journal of Statistical Software 2005; 14.

22. USAID. HIV/AIDS Health Profile: Sub-Saharan Africa - USAID; 2012. Available from: https://pdf.usaid.gov/pdf_docs/pdacu659.pdf.

23. Acharya R, Acharya T, Devkota R. Knowledge regarding prevention of mother to child transmission of HIV/AIDS among antenatal mothers. Nepal Journal of College of Medical Sciences - Nepal 2018; 14.

24. Abtew S, Awoke W, Asrat A. Knowledge of pregnant women on mother-to-child transmission of HIV, its prevention, and associated factors in Assosa town, Northwest Ethiopia. HIV/AIDS (Auckl) 2016; 8: 101-107.
25. Alemu YM, Habtewold DT, Alemu SM. Mother's knowledge on prevention of motherto-child transmission of HIV, Ethiopia: a cross sectional study. PLoS One 2018; 13: e0203043.

26. Kei MR, Ndwiga T, Okong'o S, Njoroge S. Knowledge and attitude on prevention of mother to child transmission of HIV among pregnant women attending antenatal clinic at Kisii Level Five Hospital in Kisii County, Kenya. Int J Trop Dis Health 2015; 6: 44-51.

27. Ciampa PJ, Skinner SL, Patricio SR, Rothman RL, Vermund SH, Audet CM. Comprehensive knowledge of HIV among women in rural Mozambique: development and validation of the HIV knowledge 27 scale. PLoS One 2012; 7: e48676.

28. Ciampa PJ, Vaz LME, Blevins M, et al. The association among literacy, numeracy, HIV knowledge and health-seeking behavior: a population-based survey of women in rural Mozambique. PLoS One 2012; 7: e39391.

29. Asefa A, Beyene H. Awareness and knowledge on timing of motherto-child transmission of HIV among antenatal care attending women in Southern Ethiopia: a cross sectional study. Reprod Health 2013; 10: 66 .

30. Audet CM, Sidat M, Blevins M, Moon TD, Vergara A, Vermund SH. HIV knowledge and health seeking behavior in Zambézia Province, Mozambique. SAHARA J 2012; 9: 41-46.

31. Bhagwanjee A, Govender K, Reardon C, Johnstone L, George G, Gordon S. Gendered constructions of the impact of HIV and AIDS in the context of the HIV-positive seroconcordant heterosexual relationship. J Int AIDS Soc 2013; 16: 18021.

32. Koo K, Makin JD, Forsyth BW. Where are the men? Targeting male partners in preventing mother-to-child HIV transmission. AIDS Care 2013; 25: 43-48.

33. Byamugisha R, Tumwine JK, Semiyaga N, Tylleskär T. Determinants of male involvement in the prevention of mother-to-child transmission of HIV programme in Eastern Uganda: a cross-sectional survey. Reprod Health 2010; 7: 12.

34. DeGennaro V, Zeitz P. Embracing a family-centred response to the HIV/AIDS epidemic for the elimination of pediatric AIDS. Glob Public Health 2009; 4: 386-401. 